\title{
Dimensional Analysis and Implicit Function: An Application to Textual Data
}

\author{
FERNANDO JUÁREZ \\ School of Administration \\ Universidad del Rosario \\ Autopista Norte, Calle 200, Bogotá \\ COLOMBIA
}

Abstract: - The purpose of the research is to test the viability and usefulness of the dimensional analysis

based on the Vaschy-Buckingham $\Pi$ theorem and implicit function applied to textual data. The method uses hermeneuticalanalysis that allows identifying most frequent words, phrases, and the colocations of words, defining words as categories, and then, as fundamental and derived variables; collocation textual analysis also provides the word links that create a conceptual structure to building the dimensional matrixes and equations by the Vaschy-Buckingham $\Pi$ theorem. The Gauss-Jordan method gives a solution to the matrixes. Besides, the implicit function theorem allows creating relationships among the $\Pi$ numbers and solving them by partial

derivatives, gaining insights about the relevance of variables and their relationships. As an example, the model applies to the financial summary report about Isodiol International Inc. Reports Profitable Q4 Financial Statements, delivered by EMIS. Results showed the following relevant categories:1)Company Financial Performance,2)Company,3)Continued,4)Global,5)Expand,6)Footprint,7)Diversification,8)Costs,9)Bioactive, 10 )Acquisitions,11)CBD,12)Anticipate,13)Traction,14).Additional. They were classified as fundamental and derived variables. All of them were considered derived variables, while the fundamental variables were:1)Expand,

2)Footprint,3)Diversification,4)Costs,5)Bioactive,6)Acquisitions,7)CBD,8)Anticipate,9)Traction,10). $\quad$ The application of the Vaschy-Buckingham $\Pi$ theoremresulted in four $\Pi_{\text {numbers, }}$ rearranged into an implicit function where the dependent variable was Company Financial Performance. The solution by partial derivatives resulted in identifying the category "Company Financial Performance" as well as "Traction" and "Additional" as core categories in the financial report; however, the other categories are also relevant. Conclusions point out the relevance of the analysis to textual data as an interphase between qualitative and quantitative data and also in helping to find relevant variables.

Key-Words: Dimensional Analysis; Vaschy-Buckingham Theorem; Textual Data; Implicit Function; Qualitative, Quantitative.

Received: October 2, 2019. Revised: March 20, 2020. Accepted: March 24, 2020. Published: March 31, 2020.

\section{Introduction}

The analysis of qualitative data has a rich set of new methodologies originated under a quantitative framework but promptly to enter the new field of textual data analysis, as a complex and promising interaction among methods [see 1 for an outstanding example]. Several software packs, such as ATLASTi, InVivo, MAXQDA, andVoyant Tools, offer very sophisticated methods for analyzing textual data by introducing the mentioned research mixture.

On the other hand, the profusion of methods, techniques, along with the emphasis on subjectivity, as the core of many qualitative analyses, can lead to very isolated ways of interpreting texts and very different results, depending on the preferences and viewpoint of the researcher.

While this perspective is worthy under some qualitative approaches, it might lead to unnecessary complexity and lack of agreements on many issues; i.e., in analyzing data arising from focus groups, indepth interviews, or company reports, it might be desirable some consolidated conclusions and not only a different opinion depending on the researcher's subjectivity.

Thoseare the reasons why this research tries to present another more standardized approach but based on the previous well-develop tools. To that, 
the method of dimensional analysis, widely used in other disciplines, such as physics [as an example see $2,3,4]$ and at a lesser degree in economics, statistics, artificial neural network [see 5, 6, 7], and production systems [8], is used as a paradigm to produce a standard framework; it also admits generalized $[9,10,11]$ and alternative [12] forms.

The method, which has its origin as a form of looking for dimensional homogeneity in the formulations and laws of those mentioned fields of knowledge, can be easily translated to other areas.The seminal studies of Vaschy [see 13] and Buckingham [see 14] creates the logic to interpreting and analyzing the correspondence between the two sides of an equation in terms of the dimensions involved. A detailed historical account of their validity proof is in [15].

Despite its appearance of objectivity, dimensional analysis has plenty of subjectivity, while, at the same time, presenting very objective results.However, this particular combination does not prevent the method of drawing relevant and reliable results. In this regard, it is a good candidate forcombining qualitative/quantitative data; as an example, the theorem found an application to identify some probabilistic states of the mind and ideas [see 16, 17], and also in qualitative physics [18]. Besides, the $\Pi$-theorem is more than a dimensional test for a certain field of knowledge, it is a general algebra theorem [19], and it is useful for application in any science.

\section{Problem Formulation}

The problem is the application of dimensional analysis to textual data along with elements of differential calculus to provide more analytical possibilities of combining qualitative data and quantitative analysis.

The problem arises from the inherent existing subjectivity in qualitative analysis when creating categories from textual and other qualitative data. The manner of creating those categories results in subjective structures difficult to compare with others that could be created by other researchers. In this regard, there is an opportunity to build and analyze categorical structures in a standardized reproductive way together with a different and, hopefully, productive analysis.

\section{Problem Solution}

This research is an exploratory, rational-deductive, and pragmatic one, conductive todevelop a qualitative/quantitative data analysis methodology useful to analyzing textual data that arise from qualitative research methodologies and techniques, such as focus group and in-depth interviews, but without disregarding any other type of data, such as reports, or interpretations about quantitative data, among others.

Even though the research combines qualitative and quantitative methods, the intention is not to encapsulate this combination under a specific type, but mixing the methods and data under exploratory research logic. The methods used in this research are hermeneutics and non-experimental.

As an example of the methodology, it analyzes the $20194^{\text {th }}$ quarter (Q4) financial report from IsodiolInternational Inc., a global companyinnovator in Bioactive CBD (Cannabidiol), which is a nonpsychoactive cannabinoid that occurs naturally, used for pharmaceutical and wellness products [20]. The report is located in the EMIS business database, and comprises information about profit increase and strategies used by the company to reach that increase; it contains 201 words.

There is a rising in the use of non-financial measures in the financial performance reports [21]; the assessment of financial performance admits many different tools and perspectives, among them the combinations of ratios and linguistic analysis [22], sentiment analysis to predict risk [23] and language analysis [24]. That shows an interest in other information than that in the common financial language.

The hermeneutic analysis conducted used the Voyant Tools software, which is web based-free software that gives many analytical tools to make subjective interpretations of textual data.

The hermeneutical analysis consists of many different tools to reveal the most important characteristics in textual data. One of them is the most frequent words, $F_{\mathrm{i}}$, in the text. Another one is the colocation, $F_{\mathrm{ij}}$, or frequency of closely located words. Several frequent words and colocations for the Isodiol Report are in Table 1.The table also shows the frequency change of the words throughout the text or trend.

In the table, 'company' is the most frequently located word next to others, it also is the most frequent word in the text, and so it has particular relevance in the Isodiol Report. The word itself has the meaning of self-reference related to different particular aspects of the company so that the report could be centered on that word, showing a lack of 
interest in other financial aspects not directly related to the company.

Table 1. Several of the most frequent words and colocations in the Isodiol Company Q4 Report.

\begin{tabular}{lcrllc}
\hline \multicolumn{1}{c}{ Words } & Count & Trend & \multicolumn{2}{c}{ Colocation } & Count \\
\hline Company & 6 & 0.02843 & Company & Traction & 2 \\
Continued & 3 & 0.01421 & Company & Global & 2 \\
Global & 3 & 0.01421 & Isodiol & International & 2 \\
International & 3 & 0.01421 & Global & Company & 2 \\
Isodiol & 3 & 0.01421 & Company & Strong & 2 \\
Revenues & 3 & 0.01421 & Company & Significant & 2 \\
America & 2 & 0.01421 & Company & Results & 2 \\
Continue & 2 & 0.00947 & Company & Represents & 2 \\
Distribution & 2 & 0.00947 & Company & Traction & 2 \\
\hline
\end{tabular}

Another interesting hermeneutical analysis is to obtain the most frequent phrases $F_{\mathrm{k}}$ in the text. Those for the Isodiol Report are in Table 2.

Among the frequent phrases in Table 2, one can find the word 'company' again, confirming that it has, without no doubt, a relevant meaning within the text. Due to the small number of words in the text (201), the identification of a set of words as a phrase, different to another one but repeated all along the text, also requires a small number of words; that is why the phrase length in Table two is so small (only 2-3).

Table 2. Several of the most frequent phrases in the Isodiol Company Q4 Report.

\begin{tabular}{lrrr}
\hline \multicolumn{1}{c}{ Término } & Count & Length & Trend \\
\hline Thecompany has & 2 & 3 & 2 \\
Whollyownedsubsidiaries & 2 & 3 & 2 \\
Continue to & 2 & 2 & 2 \\
Traction in & 2 & 2 & 2 \\
\hline
\end{tabular}

The analysis of the relationships among variables can use the colocation tool to identify a network of relationships. For this research, the colocation hermeneutical analysis consisted of identifying the most significant structure of words linked by proximity in the text; this structural analysis determined the proximity by creating a window of 5 pre and post words centered in every particular word, denoted as keyword, and running the window all along the text, identifying the word links.

The obtained structure of linked words was that of Figure 1.The figure answers the question $¿$ What is the financial performance of Isodiol Company inQ4?That was the purpose of the report.

The report contains several layers and tries to create a sense of hierarchy among the concepts. As the words and the concept associated with them are the most prominent in the report, that reflects the company's understanding of what the financial performance is, at least in this report.

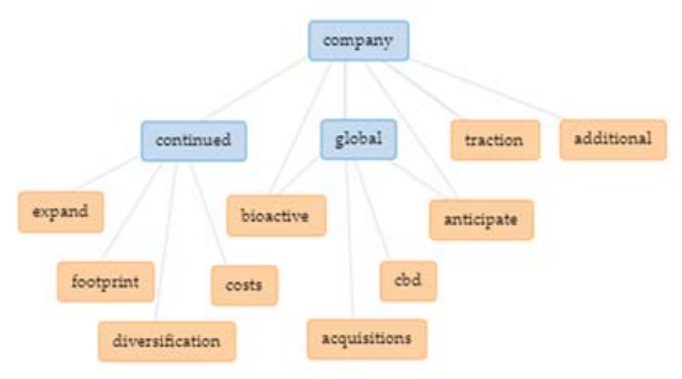

Fig. 1 The structure of colocatedwords in Isodiol financial report. The lines represent the link between words.

As shown in the figure, blue-colored squares are those words with more links to other words. Although based on link number, the figure organization is subjective and had the intention of specifically creating that structure. The viewpoint is to subjectively resemble the organization existing in other types of analysis, such as structural equation modeling or factor analysis.

The analytical procedures applied to Isodiol report were:

1. Identify categories by the hermeneutics of textual data.

2. Determining fundamentals and derived variables (categories) by analysis of the text.

3. Create and solving the dimensional matrix based on the Vaschy-Buckingham $\Pi$ theorem.

4. Apply derivation rules to the resulting equations, to identify the most relevant variables.

The interpretation of the figure is also subjective, and one can define variables as fundamentals or derived in the dimensional analysis style; such classification is subjective too, as no fundamentals or derived variables exist in textual data, except for the classification the researcher wants to create based on the textual report data. Accordingly, the following rules could apply:

1. Words are categories with meaning.

2. Categories are opinions/perceptions $=$ they are subjective.

3. As they are subjective, they have different intensity, i.e.,they have quantitative /qualitative properties.

4. Categories acquire variable characteristics.

5. Their positionin the figure could determine the type of variable, so derived variables arise from fundamental variables; the higher 
the number of arrows, the more derived the variable is, and the lesser the number of arrows, the more fundamental the variable is. However, derived variables can also be those with the lowest number of arrows, as an interpretation of them as playing a determinant role in the figure.

Following these rules and subjective criteriacould usually be part of the qualitative interpretation of categories, but in this case,the method is using logical mixed interpretations.Besides, other criteria are useful; however, the ones used in this research are fine and simple enough for clarity.

The correspondence among categories and variables is as follows:

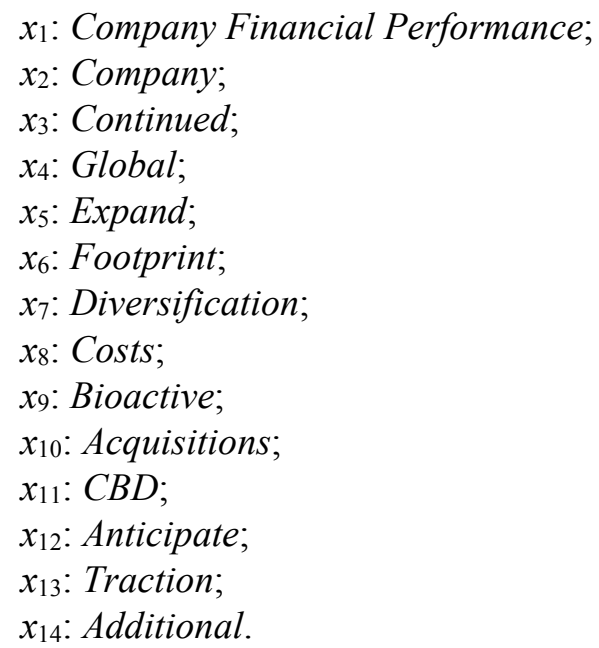

By classifying those variables into derived and fundamentals, according to the number of arrows pointing at them and their additional relevancy in the figure, derived $n$ variables are all of those previously defined, while fundamentals $k$ variables are:

\footnotetext{
$x_{5}:$ Expand;

$x_{6}$ : Footprint;

$x_{7}$ : Diversification;

$x_{8}:$ Costs;

$x_{9}$ : Bioactive;

$x_{10}$ : Acquisitions;

$x_{11}: C B D$

$x_{12}:$ Anticipate;

$x_{13}$ : Traction;

$x_{14}$ : Additional.
}

Again, the classification of a variable as derived or fundamental depends on the researcher criterion; besides, it is not unusual that a derived variable is at the same time derived and fundamental, based on its position in the relationship among variables.

The relationships among the variables are the following:
1) $x_{1}=f\left(x_{2}, x_{13}, x_{14}\right)$
2) $x_{2}=f\left(x_{3}, x_{4}, x_{13}, x_{14}\right)$
3) $x_{3}=f\left(x_{5}, x_{6}, x_{7}, x_{8}\right)$
4) $x_{4}=f\left(x_{9}, x_{10}, x_{11}, x_{12}\right)$

The analysis considers all the variables to have a power of 1; however, this criterion could change depending on the researcher's interpretation. In this case, assuming this criterion, the corresponding general dimensional matrix is in Table 1.

Table 1.Matrix with the derived variables (columns) power for each fundamental variable (rows) in the Isodiol Company Q4 Report.

\begin{tabular}{ccccccccccccccc}
\hline & $x_{5}$ & $x_{6}$ & $x_{7}$ & $x_{8}$ & $x_{9}$ & $x_{10}$ & $x_{11}$ & $x_{12}$ & $x_{13}$ & $x_{14}$ & $x_{1}$ & $x_{2}$ & $x_{3}$ & $x_{4}$ \\
\hline$x_{5}$ & 1 & 0 & 0 & 0 & 0 & 0 & 0 & 0 & 0 & 0 & 1 & 1 & 1 & 0 \\
$x_{6}$ & 0 & 1 & 0 & 0 & 0 & 0 & 0 & 0 & 0 & 0 & 1 & 1 & 1 & 0 \\
$x_{7}$ & 0 & 0 & 1 & 0 & 0 & 0 & 0 & 0 & 0 & 0 & 1 & 1 & 1 & 0 \\
$x_{8}$ & 0 & 0 & 0 & 1 & 0 & 0 & 0 & 0 & 0 & 0 & 1 & 1 & 1 & 0 \\
$x_{9}$ & 0 & 0 & 0 & 0 & 1 & 0 & 0 & 0 & 0 & 0 & 1 & 1 & 0 & 1 \\
$x_{10}$ & 0 & 0 & 0 & 0 & 0 & 1 & 0 & 0 & 0 & 0 & 1 & 1 & 0 & 1 \\
$x_{11}$ & 0 & 0 & 0 & 0 & 0 & 0 & 1 & 0 & 0 & 0 & 1 & 1 & 0 & 1 \\
$x_{12}$ & 0 & 0 & 0 & 0 & 0 & 0 & 0 & 1 & 0 & 0 & 1 & 1 & 0 & 1 \\
$x_{13}$ & 0 & 0 & 0 & 0 & 0 & 0 & 0 & 0 & 1 & 0 & 1 & 1 & 0 & 0 \\
$x_{14}$ & 0 & 0 & 0 & 0 & 0 & 0 & 0 & 0 & 0 & 1 & 1 & 1 & 0 & 0 \\
\hline
\end{tabular}

The fundamental variables in the matrixrowsare those on the lowest level of Figure 1, the ones with the lowest number of arrows pointing to them; nevertheless, they are derived variables as well, because of the interpretation made of the diagram relationshipsaccording to the previously established criteria of arrow number and relevant in the relationship among variables. According to the distribution of the fundamental and derived variable, there are n-k Пnumbers: 14-10 = 4, they are:
1) $\Pi_{1}=\left(x_{2}^{\mathrm{b}}, x_{13}{ }^{\mathrm{m}}, x_{14}{ }^{\mathrm{n}}, x_{1}^{\mathrm{a}}\right)$
2) $\Pi_{2}=\left(x_{3}{ }^{\mathrm{c}}, x_{4}{ }^{\mathrm{d}}, x_{2}{ }^{\mathrm{b}}\right)$
3) $\Pi_{3}=\left(x_{5}^{\mathrm{a}}, x_{6}{ }^{\mathrm{f}}, x_{7}^{\mathrm{g}}, x_{8}^{\mathrm{h}}, x_{3}^{\mathrm{c}}\right)$
4) $\Pi_{4}=\left(x_{9}^{\mathrm{i}}, x_{10} 0^{\mathrm{j}}, x_{11}{ }^{\mathrm{k}}, x_{12}, x_{4}^{\mathrm{d}}\right)$

Based on the $\Pi$ numbers, there are four matrixes in Table 1 , one for each $\Pi$ number. Table 2 shows the matrixes identified by their corresponding number on the first four rows in the table, the matrix numbers are those of the previously defined $\Pi$ 
numbers, i.e., $\Pi_{1}$ corresponds to Matrix 1 in the first row of the matrix rows, and the variables are those under the columns named 1 in the first matrix row.

The table equals 0 for every row, as it is the basis for $\Pi$ numbers; it is so because in every row includes derived and fundamental variables, and then, every matrix that contains a derived variable and the fundamental variables equals 0 and requires finding a vector of powers $v(\mathrm{a}, \mathrm{b}, \mathrm{c}, \ldots \mathrm{l}, \mathrm{m}, \mathrm{n})$ for each matrix $M_{\mathrm{x}}$, such as $M_{\mathrm{x}} v=0$. The column order in the matrix is important because the coefficients could have a different sign depending on that order. For instance, changing the location of the last derived variable columns to the first columns would change their sign in the equation solutions.

Table 2.Matrixes for each $\Pi$ number. Every column group with the same number in the matrix rows forms a matrix.

\begin{tabular}{|c|c|c|c|c|c|c|c|c|c|c|c|c|c|c|c|}
\hline \multicolumn{16}{|c|}{$\begin{array}{c}\text { Columns in each matrix number. Variables under the same column } \\
\text { number create a matrix. }\end{array}$} \\
\hline \multicolumn{16}{|c|}{ MR } \\
\hline$\Pi_{1}$ & 1 & 1 & 1 & 1 & 1 & 1 & 1 & 1 & 1 & 1 & 1 & & & & \\
\hline$\Pi_{2}$ & 2 & 2 & 2 & 2 & 2 & 2 & 2 & 2 & 2 & 2 & & 2 & & & \\
\hline$\Pi_{3}$ & 3 & 3 & 3 & 3 & 3 & 3 & 3 & 3 & 3 & 3 & & & 3 & & \\
\hline \multirow{2}{*}{$\Pi_{4}$} & 4 & 4 & 4 & 4 & 4 & 4 & 4 & 4 & 4 & 4 & & & & 4 & \\
\hline & $x_{5}$ & $x_{6}$ & $x_{7}$ & $x_{8}$ & $x_{9}$ & $x_{10}$ & $x_{11}$ & $x_{12}$ & $x_{13}$ & $x_{14}$ & $x_{1}$ & $x_{2}$ & $x_{3}$ & $x_{4}$ & $=$ \\
\hline \multicolumn{16}{|c|}{ VR } \\
\hline$x_{5}$ & $1 \mathrm{e}$ & 0 & 0 & 0 & 0 & 0 & 0 & 0 & 0 & 0 & $1 \mathrm{a}$ & $1 \mathrm{~b}$ & $1 \mathrm{c}$ & 0 & 0 \\
\hline$x_{6}$ & 0 & $1 \mathrm{f}$ & 0 & 0 & 0 & 0 & 0 & 0 & 0 & 0 & $1 \mathrm{a}$ & $1 \mathrm{~b}$ & $1 \mathrm{c}$ & 0 & 0 \\
\hline$x_{7}$ & 0 & 0 & $1 \mathrm{~g}$ & 0 & 0 & 0 & 0 & 0 & 0 & 0 & $1 \mathrm{a}$ & $1 \mathrm{~b}$ & $1 \mathrm{c}$ & 0 & 0 \\
\hline$x_{8}$ & 0 & 0 & 0 & $1 \mathrm{~h}$ & 0 & 0 & 0 & 0 & 0 & 0 & $1^{\mathrm{a}}$ & $1 \mathrm{~b}$ & $1 \mathrm{c}$ & 0 & 0 \\
\hline$x_{9}$ & 0 & 0 & 0 & 0 & $1 \mathrm{i}$ & 0 & 0 & 0 & 0 & 0 & $1^{\mathrm{a}}$ & $1 \mathrm{~b}$ & 0 & $1 d$ & 0 \\
\hline$x_{10}$ & 0 & 0 & 0 & 0 & 0 & $1 \mathrm{j}$ & 0 & 0 & 0 & 0 & $1^{\mathrm{a}}$ & $1 \mathrm{~b}$ & 0 & $1 d$ & 0 \\
\hline$x_{11}$ & 0 & 0 & 0 & 0 & 0 & 0 & $1 \mathrm{k}$ & 0 & 0 & 0 & $1^{\mathrm{a}}$ & $1 \mathrm{~b}$ & 0 & $1 d$ & 0 \\
\hline$x_{12}$ & 0 & 0 & 0 & 0 & 0 & 0 & 0 & 11 & 0 & 0 & $1^{\mathrm{a}}$ & $1 \mathrm{~b}$ & 0 & $1 d$ & 0 \\
\hline$x_{13}$ & 0 & 0 & 0 & 0 & 0 & 0 & 0 & 0 & $1 \mathrm{~m}$ & 0 & $1^{\mathrm{a}}$ & $1 \mathrm{~b}$ & 0 & 0 & 0 \\
\hline$x_{14}$ & 0 & 0 & 0 & 0 & 0 & 0 & 0 & 0 & 0 & 1n & $1^{\mathrm{a}}$ & $1 \mathrm{~b}$ & 0 & 0 & 0 \\
\hline MR & & & 0 & & & & & & & & & & & & \\
\hline
\end{tabular}

The relationship among the $\Pi$ numbers is:

$$
\dot{f}\left(\Pi_{1}, \Pi_{2}, \Pi_{3}, \Pi_{4}\right)=0
$$

In terms of the variables, the relationship is:

$$
f\left(\frac{x_{1}}{x_{2} \mathrm{a}, x_{13}, x_{14}}, \frac{x_{2}}{x_{3}, x_{4}}, \frac{x_{3}}{x_{5}, x_{6}, x_{7}, x_{8}}, \frac{x_{4}}{x_{9}, x_{10}, x_{11}, x_{12}}\right)=0
$$

Solving the corresponding matrix for each $\Pi$ by Gauss-Jordan elimination system requires entering in the system one derived variable power and the fundamental variable powers entered in Table 1. A combination of the variables that is a product of powers [25]. Applying the procedure, it leads to the following solutions:
1) $\Pi_{1}\left(a=x_{14} ; e=-x_{14} ; f=-x_{14} ; g=-x_{14} ; h=-x_{14} ; i=-x_{14} ; j=-\right.$ $\left.x_{14} ; k=-x_{14} ; l=-x_{14} ; m=-x_{14} ; n=-x_{14}\right)$

2) $\Pi_{2}\left(b=x_{14} ; e=-x_{14} ; f=-x_{14} ; g=-x_{14} ; h=-x_{14} ; i=-x_{14} ; j=-\right.$ $\left.x_{14} ; k=-x_{14} ; l=-x_{14} ; m=-x_{14} ; n=-x_{14}\right)$

3) $\Pi_{3}\left(c=x_{8} ; e=-x_{8} ; f=-x_{8} ; g=-x_{8} ; h=-x_{8} ; i=0 ; j=0 ; k=0\right.$; $l=0 ; m=0 ; n=0)$

4) $\Pi_{4}\left(d=x_{12} ; e=0 ; f=0 ; g=0 ; h=0 ; i=-x_{12} ; j=-x_{12} ; k=-\right.$ $x_{12} ; l=-x_{12} ; m=0 ; n=0$ )

Eliminating the $x_{\mathrm{i}}$ variables with power 0 , and substituting the others $x_{\mathrm{j}}$ with power 1 :

1) $\Pi_{1}(a=1 ; e=-1 ; f=-1 ; g=-1 ; h=-1 ; i=-1 ; j=-1 ; k=-1$; $l=-1 ; m=-1 ; n=-1)$

2) $\Pi_{2}(b=1 ; e=-1 ; f=-1 ; g=-1 ; h=-1 ; i=-1 ; j=-1 ; k=-1$; $l=-1 ; m=-1 ; n=-1)$

3) $\Pi_{3}(c=1 ; e=-1 ; f=-1 ; g=-1 ; h=-1 ; i=0 ; j=0 ; k=0$; $l=0 ; m=0 ; n=0)$

4) $\Pi_{4}(d=1 ; e=0 ; f=0 ; g=0 ; h=0 ; i=-1 ; j=-1 ; k=-1 ; l=-$ $1 ; m=0 ; n=0$ )

Reformulating the relationships among the $\Pi$ numbers in terms of the results:

$$
F\left(\frac{x_{1}}{x_{5} \ldots x_{14}}, \frac{x_{2}}{x_{5} \ldots x_{14}}, \frac{x_{3}}{x_{5} x_{6} x_{7} x_{8}}, \frac{x_{4}}{x_{9} x_{10} x_{11} x_{12}}\right)
$$

Thatrelationship agrees with the relationships among the variables depicted in Figure 1. However, according to (1), the relationship for the $\Pi$ numbers is:

$$
F\left(\frac{x_{1}}{x_{5} \ldots x_{14}}, \frac{x_{2}}{x_{5} \ldots x_{14}}, \frac{x_{3}}{x_{5} x_{6} x_{7} x_{8}}, \frac{x_{4}}{x_{9} x_{10} x_{11} x_{12}}\right)=0
$$

Now, as the relationship equals 0 , then the relationship among the $\Pi$ numbers can be defined assuming the implicit function:

$$
\frac{x_{1}}{x_{5} \ldots x_{14}}=\Phi\left(\frac{x_{2}}{x_{5} \ldots x_{14}}, \frac{x_{3}}{x_{5} x_{6} x_{7} x_{8}}, \frac{x_{4}}{x_{9} x_{10} x_{11} x_{12}}\right)
$$

In the equation, the $\Pi_{1}$ number is a function $\Phi$ of the others in such a way that (4) equals 0 . The solution for $x_{1}$ is

$$
x_{1}=x_{5} \ldots x_{14} \Phi\left(\frac{x_{2}}{x_{5} \ldots x_{14}}, \frac{x_{3}}{x_{5} x_{6} x_{7} x_{8}}, \frac{x_{4}}{x_{9} x_{10} x_{11} x_{12}}\right)
$$

However, in what follows the analysis uses the function (4), which has four variables, let them be

1) $Y=\frac{x_{1}}{x_{5} \ldots x_{14}}=x_{1} x_{5}^{-1} \ldots x_{14}{ }^{-1}$ 
2) $X_{2}=\frac{x_{2}}{x_{5} \ldots x_{14}}=x_{2} x_{5}^{-1} \ldots x_{14}^{-1}$

3) $X_{3}=\frac{x_{3}}{x_{5} x_{6} x_{7} x_{8}}=x_{3} x_{5}{ }^{-1} x_{6}{ }^{-1} x_{7}{ }^{-1} x_{8}{ }^{-1}$

4) $X_{4}=\frac{x_{4}}{x_{9} x_{10} x_{11} x_{12}}=x_{4} x_{9}^{-1} x_{10}{ }^{-1} x_{11}{ }^{-1} x_{12}{ }^{-1}$

The derivatives of the independent variables can be obtained directly:

$$
\begin{gathered}
\frac{d\left(X_{2}\right)}{d x_{2}}=\frac{d}{d x_{i}}\left[\frac{x_{2}}{x_{5} \ldots x_{14}}\right]=\frac{1}{x_{5} \ldots x_{14}} \\
\frac{d\left(X_{3}\right)}{d x_{3}}=\frac{d}{d x_{i}}\left[\frac{x_{3}}{x_{5} x_{6} x_{7} x_{8}}\right]=\frac{1}{x_{5} x_{6} x_{7} x_{8}} \\
\frac{d\left(X_{4}\right)}{d x_{4}}=\frac{d}{d x_{i}}\left[\frac{x_{4}}{x_{9} x_{10} x_{11} x_{12}}\right]=\frac{1}{x_{9} x_{10} x_{11} x_{12}}
\end{gathered}
$$

Any variable $x_{\mathrm{i}}$ could be the differentiation variable, i.e., one of the denominator variables as well. However, to keep the relevance of the fundamental variables derived variables were the differentiation variablesonly.

Obtaining the dependent variable derivatives requires a different procedure; it needs to take partial derivatives for $Y$ (dependent variable) and each $X_{i}$ (independent variables). The computations are

$$
\begin{aligned}
& \frac{\partial f}{\partial X_{2}}=-\frac{F_{X 2}^{\prime}}{F_{Y}^{\prime}} \\
& \frac{\partial f}{\partial X_{3}}=-\frac{F_{X 3}^{\prime}}{F_{Y}^{\prime}} \\
& \frac{\partial f}{\partial X_{4}}=-\frac{F_{X 4}^{\prime}}{F_{Y}^{\prime}}
\end{aligned}
$$

Results are

$$
\begin{gathered}
\frac{\partial f}{\partial X_{2}}=\frac{F_{X 2}^{\prime}}{F_{Y}^{\prime}}=-\frac{\frac{1}{x_{5} \ldots x_{14}}}{\frac{1}{x_{5} \ldots x_{14}}}=-1 \\
\frac{\partial f}{\partial X_{3}}=\frac{F_{X 3}^{\prime}}{F_{Y}^{\prime}}=-\frac{\frac{1}{x_{5} x_{6} x_{7} x_{8}}}{\frac{1}{x_{5} \ldots x_{14}}} \\
=-x_{9} x_{10} x_{11} x_{12} x_{13} x_{14}
\end{gathered}
$$

$$
\begin{aligned}
\frac{\partial f}{\partial X_{4}} & =\frac{F_{X 4}^{\prime}}{F_{Y}^{\prime}}=-\frac{\frac{1}{x_{9} x_{10} x_{11} x_{12}}}{\frac{1}{x_{5} \ldots x_{14}}} \\
& =-x_{5} x_{6} x_{7} x_{8} x_{13} x_{14}
\end{aligned}
$$

In the equations (13), (14) and (15), the independent variable $\left(X_{2}, X_{3}, X_{4}\right)$ derivatives are those in (7), (8), and (9) respectively.

Although results are somehow expected, due to the subjective designation of power 1 on each variable, there are the following conclusions:

1) Despite the assertion that $x_{1} \neq x_{2}$, results show (equation 13) that increments in $Y$ are the same as $X_{2}$ because variations in the same variables cause them; so then, they confirm the strong relationship between $x_{1}$ and $x_{2}$; actually, $x_{2}$ can be the direct answer to the main question $\left(x_{1}\right)$. In this regard, the identified core message of the report is the company itself.

2) The results agree with the hierarchy depicted in Figure 1, showing the relevance of each derived and fundamental variable (equations 13, 14, 15).

3) The resultspoint out the relevance of variables $x_{13}$ and $x_{14}$, as they have a direct effect on $Y$ (equations 13, 14). That shows how several elementary items have an important effect on the financial performance report.

4) It consolidates the results intuitively obtained by looking at Figure 1. In this sense, it is a double check of the model and the results.

Finally, the analysis is also useful to identify those values of the company's financial status by looking at those items that proved to be most relevant in the results.

The analysis of the Isodiol report is an example of how this mathematical analysis could help in providing a different insight into textual data. Despite the briefness of the report and the subjective application of powers to variables, the analysis shows many possibilities in their applications in more complex texts. 


\section{Conclusion}

This research shows the analysis for qualitative data that complement those already existing by introducing a new method applied to thereof. It provides new insights into the possibilities that a mixture of quantitative/qualitative data has.

The method constitutes a methodological integration that goes further than a mix of quantitative and qualitative research. The research intentionally avoided that type of methodological classification regarding the method; however, data are textual or numerical, and, in that sense, qualitative/quantitative denominations are perfectly right.

The method introduced here also allows comparing data within a standard analysis framework, and the application to simultaneous analysis of different texts is easy.

The text, type of variables, and their relationships used in this example have the intention of showing the possibilities of the analysis; however, it admits many levels of complexity, depending on the research objectives and the researcher's subjective consideration about data.

Some useful studies relevant tothis research can be found in[26], [27], and [28].

\section{References:}

[1] Manning, Ch. D., \&Schuze, H., FoundationsofStatisticalNaturalLanguageP rocessing, Cambridge, Massachusetts: The MIT Press, 1999.

[2] Sonin, A. A., The Physical Basis of Dimensional Analysis ( $2^{\text {nd }}$ ed.), Cambridge, Massachusetts: The MIT Press, 2001.

[3] Lira, I., Dimensional analysis made simple, European Journal of Physics, Vol. 34, 2013, pp. 1391-1401.

[4] Albrecht, M., Nachtsheim, C., Albrecht, T., \& Cook, R. D. Experimental Design for Engineering Dimensional Analysis. Technometrics, Vol. 55, No. 3, 2013, pp. 257-270.

[5] Poveda, G., El análisis dimensional en economía, Revista Soluciones de Postgrado EIA, Vol. 12, 2014, pp. 67-94.

[6] Weijie, D. T., Lin, D. K. J., \&Nachtsheim, C. J. Dimensional Analysis and Its Applications in Statistics. Journal of Quality Technology, Vol. 46, No. 3, 2014, pp.185-198.

[7] Phate, M. R., \& Toney, S. B. Modeling and prediction of WEDM performance parameters for $\mathrm{Al} / \mathrm{SiCp} \mathrm{MMC}$ using dimensional analysis and artificial neural network. Engineering Science and Technology, an International Journal, Vol. 22, No. 2, 2019, pp. 468-476.

[8] Miragliotta, G. The power of dimensional analysis in production systems design. International Journal of Production Economics, Vol. 131, No. 1, 2011,pp.175182.

[9] Ain A. Sonin, \& Ronald F. Probstein. A Generalization of the II-Theorem and Dimensional Analysis. Proceedings of the National Academy of Sciences of the United States of America, Vol. 101, No. 23, 2004, pp. 8525.

[10] Poveda, G. Análisis Dimensional Generalizado. Revista EIA, Vol. 13, No. 25, 2016, pp. 13-27.

[11] Hainzl, J. On local generalizations of the pi-theorem of dimensional analysis. Journal of the Franklin Institute, Vol., 292, No. 6, (n.d.), pp. 463-470.

[12] Pankhurst, R. C. Alternative formulation of the pi-theorem. Journal of the Franklin Institute, Vol. 292, No.6, (n.d.),pp. 451462.

[13] Vaschy, A. Sur les lois de similitude en physique. AnnalesTélégraphiques, Vol. 19, 1892, pp. 25-28.

[14] Buckingham, E.On physically similar systems. Illustrations of the use of dimensional equations. Physical Review, Vol. 4, 1914, pp. 345-376.

[15] Pobedrya, B. E., \&Georgievskii, D. V. On the proof of the $\Pi$-theorem in dimension theory. Russian Journal of Mathematical Physics, Vol. 13, No. 4, (2006). pp. 431437.

[16] Martínez, A., Pando, V., del Río, J. Generalizaciones al teorema $\mathrm{Pi} \mathrm{de}$ Buckingham con algunas aplicaciones. Technical Report, 2007. Retrieved from http://www.oasification.com/archivos/Pideb uck.pdf

[17] Martínez, A., Pando, V., del Río, J. Del Teorema $\pi$ al axioma de las conjeturas razonadas.Boletín de la Sociedad Puig Adam, No. 86, 2010, pp. 55-73.

[18] Bhaskar, R., Nigam, A. Qualitative physics using dimensional analysis. Artificial Intelligence, Vol. 45, No. 1-2, 1990, pp. 73-111.

[19] Curtis, W., Logan,J., Parker. W. Dimensional analysis and the $\Pi$-theorem, 
Linear Algebra and its Applications, Vol. 47, 1982, pp. 117-126.

[20] Isodiol. What is CBD. (n.d.) Retrieved from https://isodiol.com/productcategory/brand/bioactive/

[21] Muehlbacher, J., Siebenaler, T., $\&$ Wurflingsdobler, $U$. The rise of nonfinancial performance measures in annual reports. An analysis of ATX-listed companies. Trendy Ekonomiky a Management, Vol. 25, No. 9, 2016, pp. 9-21

[22] Myskova, R., \& Hajek, P. Comprehensive Assessment of Firm Financial Performance Using Financial Ratios and Linguistics Analysis of Annual Reports. Journal of International Studies, Vol. 10, No. 4, 2017, pp. 96-108.

[23] Tsai, M. F., \& Wang, C.J. On the Risk Prediction and Analysis of Soft Information in Finance Reports. European Journal of Operational Research, Vol. 257, No. 1, 2017, pp. 243-250.

[24] Slattery, D. M. The Language of Financial Reports and News. Journal of Technical Writing \& Communication, Vol. 45, No. 1, 2015, pp. 77-94.

[25] Hanche-Olsen, H. Buckingham's pitheorem. TMA4195 Mathematical modeling. $2004 . \quad$ Retrieved from http://folk.ntnu.no/hanche/notes/buckingha $\mathrm{m} /$ buckingham-a4.pdf

[26] Jothi, V., \&Nithya, L.M.Semi-supervised Taxonomy Aware Integration of Catalogs. WSEAS Transactions on Information Science and applications. Vol. 11, 2014, pp. 169-176.

[27] Ghazarian, A. A Dimension-Oriented Theory of Requirements Space in BusinessInformation Systems. WSEAS Transactions on Systems. Vol. 13, 2014, pp. 76-95.

[28] Skala, V. Fast Interpolation and Approximation of Scattered Multidimensional and Dynamic Data Using Radial Basis Functions. WSEAS Transactions on Mathematics. Vol. 12, No. 5, 2013, pp. 501-511. 